Originally published as:

Koch, J., Dworak, R., Prager, R., Becker, B., Brockmann, S., Wicke, A., Wichmann-Schauer, H., Hof, H., Werber, D., Stark, K.

Large listeriosis outbreak linked to cheese made from pasteurized milk, Germany, 2006-2007 (2010) Foodborne Pathogens and Disease, 7 (12), pp. 1581-1584.

DOI: 10.1089/fpd.2010.0631

This is a copy of an article published in the Foodborne Pathogens and Disease (C) 2010 copyright Mary Ann Liebert, Inc.;

Foodborne Pathogens and Disease is available online at: http://online.liebertpub.com 


\title{
Large Listeriosis Outbreak Linked to Cheese Made from Pasteurized Milk, Germany, 2006-2007
}

\author{
Judith Koch, ${ }^{1}$ Regine Dworak, ${ }^{2}$ Rita Prager, ${ }^{3}$ Biserka Becker, ${ }^{4}$ Stefan Brockmann, ${ }^{5}$ Amal Wicke, ${ }^{6}$ Heidi \\ Wichmann-Schauer, ${ }^{7}$ Herbert Hof, ${ }^{8}$ Dirk Werber, ${ }^{1}$ and Klaus Stark ${ }^{1}$ \\ ${ }^{1}$ Department for Infectious Disease Epidemiology, Robert Koch Institute, Berlin, Germany. \\ ${ }^{2}$ Public Health Office, Ploen, Germany. \\ ${ }^{3}$ National Reference Center for Salmonella and Other Bacterial Enteric Pathogens, Robert Koch \\ Institute, Wernigerode, Germany. \\ ${ }^{4}$ Federal Research Institute of Nutrition and Food, Max Rubner Institute, Karlsruhe, Germany. \\ ${ }^{5}$ Baden-Württemberg State Health Office, Department of Epidemiology and Health Reporting, \\ Stuttgart, Germany. \\ ${ }^{6}$ Consumer Protection Office, Halle, Germany. \\ ${ }^{7}$ Federal Institute for Risk Assessment, Berlin, Germany. \\ ${ }^{8}$ Reference Laboratory for Listeriosis (until 2009), University of Heidelberg, Heidelberg, Germany.
}

Address correspondence to:

Klaus Stark, M.D., Ph.D., M.Sc. (Epid.)

Department for Infectious Disease Epidemiology

Robert Koch Institute

DGZ-Ring 1

13086 Berlin

Germany

E-mail:starkk@rki.de

\begin{abstract}
A commercial cheese (acid curd) made from pasteurized milk caused a large listeriosis outbreak in Germany from October 2006 through February 2007. The Listeria monocytogenes outbreak strain was identified in humans and in cheese samples from a patient's home and from the production plant. During the outbreak period, 189 patients were affected, which was $97 \%$ above the mean case number for the respective time period of the years 2002 to 2005. Of patients with available detailed information on cheese consumption ( $n=47), 70 \%$ reported to have consumed the incriminated cheese product. Recent European food safety alerts due to Listeria-contaminated cheeses more often concerned products made from pasteurized or heat-treated milk than from raw milk. The findings should be considered in prevention guidelines addressing vulnerable populations.
\end{abstract}

\section{Introduction}

Infection by Listeria monocytogenes can lead to severe illness, including sepsis, meningitis, stillbirth, and death. Risk groups for listeriosis are immunocompromised and elderly individuals, and pregnant women. In Germany, notification of invasive L. monocytogenes infections is mandatory. Annually 300 to 500 patients are notified (Koch and Stark, 2006). In recent years, an increase of listeriosis cases including larger outbreaks has been observed in several countries (Denny and McLauchlin, 2008).

In general, listeriosis is a foodborne disease and vehicles characteristically are processed refrigerated foods that are consumed without further cooking, for example, meat products, seafood, and dairy products (Schlech et al., 1983). Especially unpasteurized dairy products may carry a markedly elevated risk and it is recommended that vulnerable individuals refrain from consuming such products. Little is known about risks associated with products made from pasteurized milk. We report on a nationwide listeriosis outbreak in the year 2006/2007 that was linked to a commercial acid curd cheese made from pasteurized milk. 


\section{Materials and Methods}

In Germany, isolation of L. monocytogenes from a normally sterile substrate (e.g., blood and cerebrospinal fluid) or from any specimen in neonates is mandatorily notifiable in the national surveillance. The clinical case definition is fulfilled if in addition to the laboratory confirmation specified clinical characteristics are reported (i.e., meningitis, septicemia, still birth, or fetal loss).

On 21st November 2006, a 71-year-old woman with chronic alcoholic liver cirrhosis died from $L$. monocytogenes septicemia and was notified to the local public health department on 24th November. L. monocytogenes was isolated in high concentrations $\left(5.2 \times 10^{4}-1.2 \times 10^{5} \mathrm{cfu} / \mathrm{g}\right)$ from three unopened acid curd cheese samples from the patient's refrigerator, another similar cheese had been consumed by the patient. A public warning regarding particular brands of the cheese product was issued on 29th November. After $L$. monocytogenes had been also cultured from cheese samples retained at the production plant, the manufacturer announced an open recall of the cheese lot on 1st December. The implicated cheese product ("Harzer Käse") is a soft-textured cheese made from pasteurized milk that is curdled by the addition of lactic acid bacteria and ripened with a red smear (e.g., Brevibacterium linens). The cheese generally ripens from the outside to the center for no more than 2 weeks in comparative warmth. It has $<10 \%$ fat, a low dry matter $(32 \%-45 \%)$, and a high moisture content facilitating microbial growth.

We conducted an investigation to assess whether the incriminated cheese product had caused additional listeriosis cases, and to assess the scope of the outbreak. Further, we asked the German Federal Office of Consumer Protection and Food Safety about notifications of Listeria-contaminated cheese that were distributed in Europe by the Rapid Alert System for Food and Feed (RASFF) operated by the European Commission (http://ec.europa.eu/food/food/rapidalert/index_en.htm).

Local public health departments inquired case patients with disease onset during the outbreak period whether they had consumed cheese of the specified brands during the 4-week period (based on a median incubation period of about 3 weeks) before disease onset. L. monocytogenes strains were isolated from food and human samples according to international standards (International Organization for Standardization [ISO], Standard 11290). Agar Listeria selon Ottaviani \& Agosti (ALOA) chromogenic agar (AES Laboratoire, Angelbachtal, Germany) and polymyxin acriflavine lithium-chloride ceftazidime aesculin mannitol (PALCAM)-Listeria-selective agar (VWR International, Bruchsal, Germany) were used as culture plates. For serotyping we used a multiplex polymerase chain reaction assay based on the $L$. monocytogenes marker genes Imo 737, Imo 1118, ORF 2110, and ORF 2819, as proposed by Doumith et al. (2004). This polymerase chain reaction assay separates the four major serovars $(1 / 2 \mathrm{a}, 1 / 2 \mathrm{~b}, 1 / 2 \mathrm{c}$, and $4 \mathrm{~b})$ of pathogenic $L$. monocytogenes into distinct groups. Pulsed-field gel electrophoresis (PFGE) was used for molecular subtyping of isolates following the standardized PulseNet protocol (Hunter et al., 2005).

We considered the maximum outbreak period ranging from 30th October 2006 (calendar week 44/2006) to 18th February 2007 (calendar week 7/2007), based on the manufacturing date of the implicated cheese (22nd October 2006/calendar week 43), a few days of product distribution, and the "best before" date (8th December 2006/week 49), assuming an incubation period of 3-70 days.

\section{Results}

Overall, 189 patients with disease onset during the maximum outbreak period were reported, an excess of $97 \%$ compared to the mean case number $(n=96$, range $85-108)$ for the respective time period of the years 2002 to 2005 (Fig. 1). Overall, $81 \%$ of the patients were hospitalized. The case fatality rate was $14 \%$. Notified cases resided in 13 of the 16 German federal states. The median age was 69 years, and $62 \%$ were men. There were 11 pregnant women of whom 7 (64\%) suffered from pretime delivery. For $62 \%$ of the notified cases underlying immunocompromising medical conditions were reported.

Serotyping information was available for $37(20 \%)$ L. monocytogenes cases. Of these, 30 isolates were serovar $4 b$, as were the cheese isolates. The remaining 7 human isolates were serovar $1 / 2 a$ $(n=5)$ and serovar $1 / 2 \mathrm{~b}(n=2)$. An indistinguishable PFGE pattern (outbreak pattern) was found in 14 of 16 human serovar $4 \mathrm{~b}$ isolates available for molecular typing (Fig. 2). The outbreak pattern was also 
detected in the Listeria isolates from all 7 contaminated cheese samples ( 3 unopened leftovers from index household and 4 retain samples from the plant).

For 47 notified cases, information about cheese consumption was available. Of these, 34 persons $(70 \%)$ reported to have consumed the implicated cheese product. Of the eight cases with information on both cheese consumption and subtyping, five cases had consumed the implicated cheese and their isolate had the outbreak PFGE pattern or (if this was not available) was serovar 4b. By contrast, the isolates of the three case-patients who had not eaten the cheese had a non-outbreak PFGE pattern $(p=0.018)$.

All Listeria-positive cheese samples were from identical lots with the same production and "best before" date, but were sold under different brand names. In one of the 60 environmental samples from the production plant, L. monocytogenes was detected (not available for PFGE). Food safety authorities recommended measures to the manufacturer to prevent $L$. monocytogenes contamination of products during the manufacturing process, such as an improved hygiene regime guided by a Hazard Analysis and Critical Control Point system and distribution of the product conditional on Listeria-negative testing.

From 1st January 2004 to 17th September 2008, 58 alert notifications about cheeses, mainly soft cheeses, contaminated with $L$. monocytogenes were distributed in Europe by the RASFF. Of these, 10 concerned raw milk cheeses and 22 (diverse) cheese products usually made from pasteurized or heattreated milk $\left(>71^{\circ} \mathrm{C}\right)$. For the remaining 26 notifications, the treatment of the milk could not be specified.

\section{Discussion}

This was the largest recognized listeriosis outbreak in Germany, linked to a highly contaminated commercial acid curd cheese made from pasteurized milk. Evidence came from molecular typing of Listeria isolates from patients and from cheese products. The majority of cases with available specific epidemiological or microbiological information were linked to the outbreak. This outbreak, typically for listeriosis, was widely dispersed and lasted several weeks reflecting the long incubation period and long shelf life. Yet, in the absence of a routine molecular typing surveillance for human Listeria isolates, as successfully implemented in some countries (de Valk et al., 2005; Gerner-Smidt et al., 2006), our outbreak investigation was largely retrospective.

Listeria is killed by pasteurization (Lovett 1988) and outbreaks have rarely been associated with pasteurized milk products, including cheese (Lyytikainen et al., 2000; CDC, 2001). However, since the pathogen is ubiquitous, contamination may occur subsequent to the heat treatment, which usually precedes the actual cheese production. Contaminated soft-textured cheeses are particularly prone to bacterial growth. $L$. monocytogenes has been detected in cheeses made from pasteurized milk in food monitoring (Rudolf and Scherer, 2001). Further, a substantial proportion of European food alerts due to Listeria-contaminated cheeses concerned various cheese products made from pasteurized milk. In the same line of evidence is a recent listeriosis outbreak in Austria and Germany that was also caused by an acid curd cheese (Fretz et al., 2010).

Continued regulatory and industry efforts are needed to decrease Listeria in foods, including pasteurized products. Soft-textured cheeses, including acid curd cheeses labeled as made from pasteurized milk, may erroneously invoke a feeling of safety to populations vulnerable for listeriosis, and these groups need to be appropriately educated.

\section{Acknowledgments}

Günter Sommerfeld and Jan Hoffbauer, Federal Institute of Consumer Protection and Food, are thanked for providing the RASFF notification data on Listeria-contaminated cheese. 


\section{Disclosure Statement}

No competing financial interests exist.

\section{References}

[CDC] Centers for Disease Control and Prevention. Outbreak of listeriosis associated with homemade Mexican-style cheese-North Carolina, October 2000-January 2001. MMWR Morb Mortal Wkly Rep 2001;50:560-562.

Denny $\mathrm{J}$ and McLauchlin J. Human Listeria monocytogenes infections in Europe-an opportunity for improved European surveillance. Euro Surveill 2008;13:pii: 8082.

de Valk H, Jacquet C, Goulet V, Vaillant V, Perra A, Simon F, Desenclos JC, and Martin P.

Surveillance of listeria infections in Europe. Euro Surveill 2005;10:251-255.

Doumith M, Buchrieser C, Glaser P, Jacquet C, and Martin P. Differentiation of the major Listeria monocytogenes serovars by multiplex PCR. J Clin Microbiol 2004;42:3819-3822.

Fretz R, Sagel U, Ruppitsch W, Pietzka AT, Stöger A, Huhulescu S, Heuberger S, Pichler J, Much P, Pfaff G, Stark K, Prager R, Flieger A, Feenstra O, and Allerberger F. Listeriosis outbreak caused by acid curd cheese "Quargel," Austria and Germany 2009. Euro Surveill 2010;15:pii: 19477.

Gerner-Smidt P, Hise K, Kincaid J, Hunter S, Rolando S, Hyytia-Trees E, Ribot EM, Swaminathan B, and PulseNet Taskforce. PulseNet USA: a five-year update. Foodborne Pathog Dis 2006;3:9-19. Hunter SB, Vauterin P, Lambert-Fair MA, Van Duyne MS, Kubota K, Graves L, Wrigley D, Barrett T, and Ribot E. Establishment of a universal size standard strain for use with the PulseNet standardized pulsed-field gel electrophoresis protocols: converting the national databases to the new size standard. J Clin Microbiol 2005;43:1045-1050.

Koch J and Stark K. Significant increase of listeriosis in Germany-epidemiological patterns 20012005. Euro Surveill 2006;11:85-88.

Lovett J. Isolation and identification of Listeria monocytogenes in dairy products. J Assoc Off Anal Chem 1988;71:658-660.

Lyytikainen O, Autio T, Maijala R, Ruutu P, Honkanen-Buzalski T, Miettinen M, Hatakka M, Mikkola J, Anttila VJ, Johansson T, Rantala L, Aalto T, Korkeala H, and Siitonen A. An outbreak of Listeria monocytogenes serotype 3a infections from butter in Finland. J Infect Dis 2000;181:1838-1841.

Rudolf $\mathrm{M}$ and Scherer S. High incidence of Listeria monocytogenes in European red smear cheese. Int J Food Microbiol 2001;63:91-98.

Schlech WF III, Lavigne PM, Bortolussi RA, Allen AC, Haldane EV, Wort AJ, Hightower AW, Johnson SE, King SH, Nicholls ES, and Broome CV. Epidemic listeriosis-evidence for transmission by food. N Engl J Med 1983;308:203-206. 


\section{Figures}

Figure 1. Reported listeriosis cases by week of disease onset (week 36/2006-week 10/2007) with maximum outbreak period ranging from week 44 (2006) to week 7 (2007).

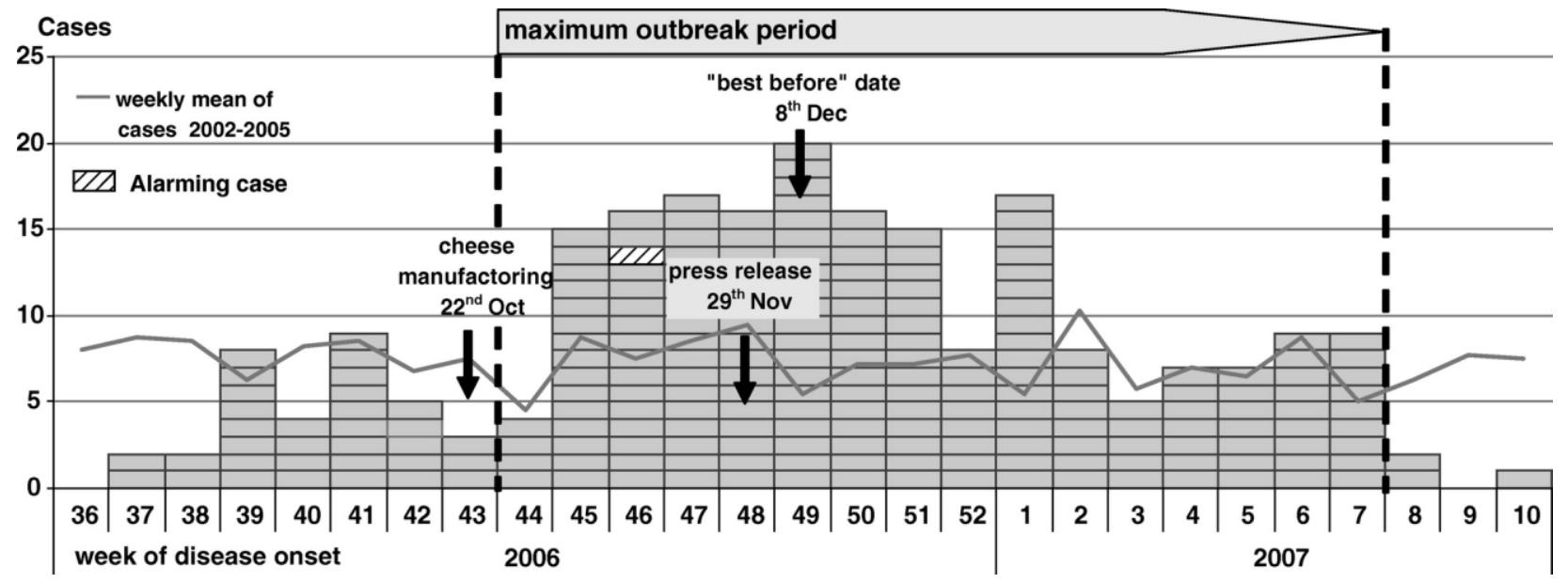

Figure 2. Ascl patterns of selected Listeria monocytogenes isolates (lanes 1 to 3 refer to $L$. monocytogenes isolates serotype $4 \mathrm{~b}$ obtained from patients before the outbreak; all other lanes refer to isolates obtained during the outbreak period; lanes 5 to 14 show the outbreak pattern).

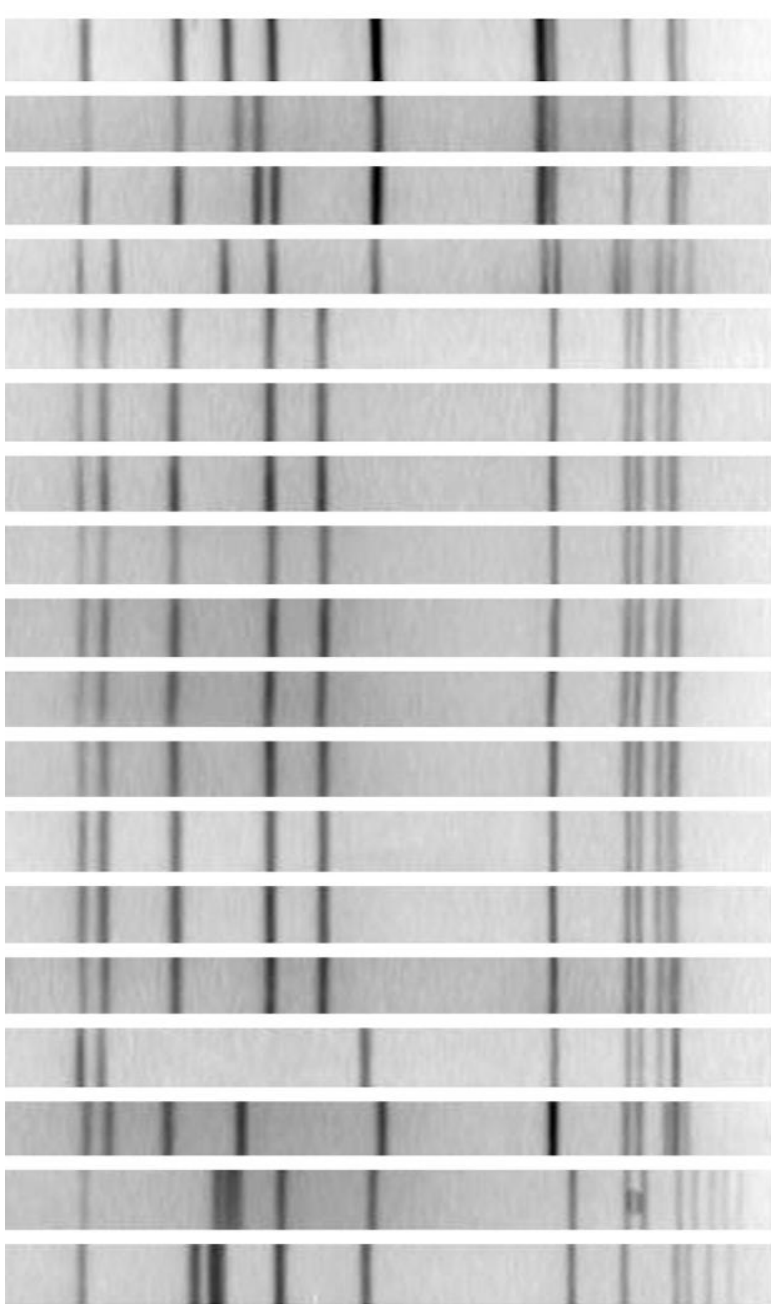

\section{Serotype Source}

$4 \mathrm{~b}$

$4 b$

$4 b$

1

$4 b$

$4 b$

$4 \mathrm{~b}$

$4 b$

$4 b$

$4 b$

$4 b$

$4 \mathrm{~b}$

$4 b$

$4 b$

$1 / 2 a$

$1 / 2 a$ human

human

human

human

cheese

cheese

cheese

human

human

human

human

human

human

human

human

human

human

human 